\title{
Interventional cardiology in Poland in 2019. Summary report of the Association of Cardiovascular Interventions of the Polish Cardiac Society (AISN PTK) and Jagiellonian University Medical College*
}

\author{
Dariusz Dudek ${ }^{1}$, Zbigniew Siudak², Marek Grygier ${ }^{3}$, Aleksander Araszkiewicz $^{3}$, Maciej Dąbrowski $^{4}$, \\ Jacek Kusa ${ }^{5}$, Michał Hawranek ${ }^{6}$, Zenon Huczek ${ }^{7}$, Paweł Kralisz ${ }^{8}$, Tomasz Roleder ${ }^{9}$, Wojciech Wojakowski ${ }^{10}$, \\ Radosław Parma ${ }^{10}$, Krzysztof P. Malinowski ${ }^{1}$, Stanisław Bartuś ${ }^{1}$
}

\begin{abstract}
${ }^{1}$ Institute of Cardiology, Jagiellonian University Medical College, Krakow, Poland
${ }^{2}$ Collegium Medicum, Jan Kochanowski University, Kielce, Poland

${ }^{3} 1^{\text {st }}$ Department of Cardiology, Poznan University of Medical Sciences, Poznan, Poland

${ }^{4}$ Department of Interventional Cardiology and Angiology, Institute of Cardiology, Warsaw, Poland

${ }^{5}$ Pediatric Cardiology Department, Regional Specialist Hospital - Research and Developement Centre, Wroclaw, Poland

${ }^{6} 3^{\text {rd }}$ Department of Cardiology, Faculty of Medical Sciences in Zabrze, Medical University of Silesia in Katowice, Poland

${ }^{7} 1^{\text {st }}$ Department of Cardiology, Medical University of Warsaw, Warsaw, Poland

${ }^{8}$ Department of Invasive Cardiology, Medical University of Bialystok, Bialystok, Poland

${ }^{9}$ Regional Specialist Hospital, Research and Development Center, Wroclaw, Poland

${ }^{10}$ Department of Cardiology and Structural Heart Diseases, $3^{\text {rd }}$ Division of Cardiology, Medical University of Silesia, Katowice, Poland

*This publication presents an analysis of individual procedural data from 153 interventional cardiology centers in Poland that have voluntarily joined the ORPKI database since 2004. To account for possible underreporting observed in 2019, AISN PTK makes every effort to correct for missing data and provide reliable information on interventional cardiology in Poland.
\end{abstract}

Adv Interv Cardiol 2020; 16, 2 (60): 123-126 DOI: https://doi.org/10.5114/aic.2020.96054

As previously reported, the ORPKI electronic data capture is monitored continuously and modified according to the needs of the rapidly changing everyday practice of interventional cardiology not only in Poland but also worldwide [1, 2]. The database is endorsed by Association of Cardiovascular Interventions of the Polish Cardiac Society (AISN PTK) and is operated by the Jagiellonian University Medical College, with currently 153 interventional cardiology centers in Poland reporting daily. The ORPKI database analysis of various trends and patterns is published each year [3-5]. On 31 ${ }^{\text {st }}$ of December 2019 there were 541 percutaneous coronary intervention (PCI) operators and 32 diagnosticians certified by AISN PTK in Poland.

According to the current analysis of the ORPKI database, in comparison to 2018 , there was a substantial decrease in the total number of reported coronary angiographies (CAG) [1]. There were 172521 CAG [(4481 per 1 million inhabitants per year) in 2019, which corresponds to a decrease of $5.5 \%$ vs. 2018 (Figure 1). This trend has been observed since 2015, and current numbers are comparable to that of the year 2009. The distribution of primary diagnosis as indications for CAG was: 11\% ST-elevation myocardial infarction (STEMI), 14\% non-ST-elevation myocardial infarction (NSTEMI), 26\% unstable angina (UA), and $49 \%$ stable angina. The radial approach was utilized in $88 \%$ of all CAG cases, which is a $2 \%$ rise in comparison to 2018 (in STEMI patients, 85\% had the radial access site used). Complications of coronary angiography in 2019 were rare and corresponded to the rates reported in previous years (Table I).

The total number of $\mathrm{PCl}$ procedures was 101716 and is $2.9 \%$ lower (2642 $\mathrm{PCls}$ per 1 million inhabitants per year) than that reported to the ORPKI database in 2018 (Figure 1). The majority of the procedures were done in the setting of the acute coronary syndromes (ACS): $40 \%$ acute myocardial infarction (20\% STEMI and $20 \%$ NSTEMI), $24 \%$ unstable angina (decrease by $2 \%$ from

Corresponding author:

Zbigniew Siudak MD, PhD, Collegium Medicum, Jan Kochanowski University, 19 IX Wieków Kielc St, 25-516 Kielce, Poland,

e-mail: zbigniew.siudak@ujk.edu.pl

Received: 10.05.2020, accepted: 11.05.2020. 

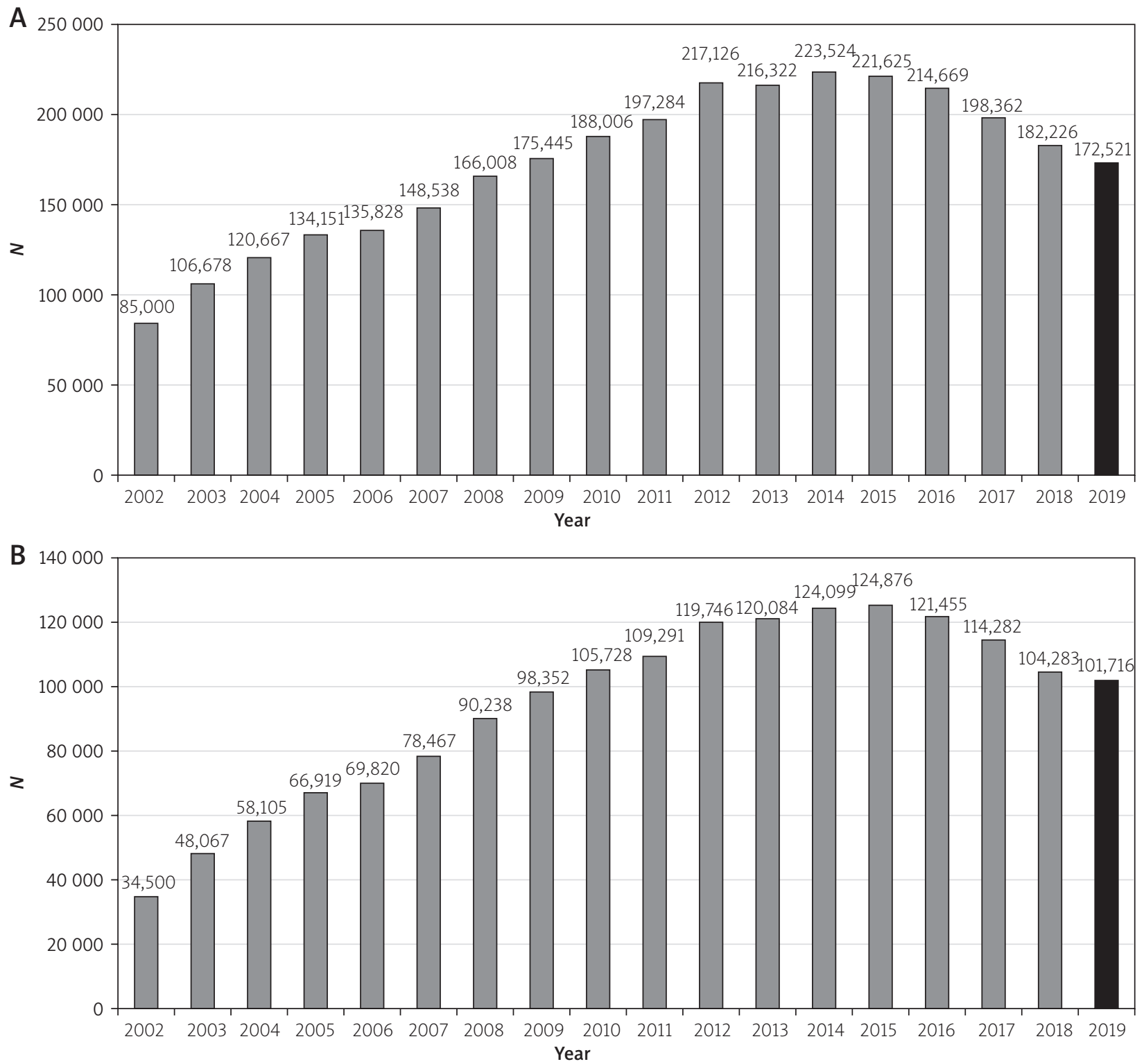

Figure 1. The number of coronary angiography (A) and PCI (B) procedures in Poland in the years 2002-2019

2018) and the remaining $36 \%$ for stable angina. The number of primary $\mathrm{PCl}$ per 1 million inhabitants per year is currently 505. There were $19440 \mathrm{PCIS} \mathrm{in} \mathrm{STEMI}$ and 20078 in NSTEMI. Drug-eluting stents (DES) are used in $99.9 \%$ of all cases, while bioresorbable vas-

Table I. Complications of coronary angiography in Poland in 2019

\begin{tabular}{lcc} 
Parameter & $\%$ & In comparison to 2018 \\
\hline Death & 0.20 & $\uparrow$ \\
\hline Stroke & 0.01 & $\leftrightarrow$ \\
\hline Major bleeding at access site & 0.04 & $\leftrightarrow$ \\
\hline Sudden cardiac arrest & 0.18 & $\leftrightarrow$ \\
\hline Allergic reaction & 0.01 & $\downarrow$
\end{tabular}

cular scaffold (BVS) were implanted in 66 procedures only. Rotablation was used in 1172 procedures $(1.1 \%$ of $\mathrm{PCI}$ - a $38 \%$ increase in comparison to the previous year. Aspiration thrombectomy was used in only 1845 STEMI cases, which means a $26 \%$ decrease in comparison to 2018. We observed a substantial increase in the use of guideline-recommended ticagrelor as an adjunct pharmacotherapy both for STEMI (49\%) and NSTEMI (30\%) with the use of prasugrel less than $2 \%$ in either indication. In terms of complex procedures, the number of chronic total occlusion (CTO) procedures was 2299. PCl complications are still rare and are presented in Table II.

An increase in the use of adjunctive imaging and diagnostic procedures during CAG and PCI was observed in 2019 vs. 2018, especially for intravascular ultrasound (IVUS) (Table III). 
Table II. Complications of PCI in Poland in 2019

\begin{tabular}{llc} 
Parameter & $\%$ & $\begin{array}{c}\text { In comparison to } \\
2018\end{array}$ \\
\hline Death: & 0.32 & $\downarrow$ \\
\hline STEMI & 0.83 & \\
\hline NSTEMI & 0.36 & \\
\hline UA & 0.10 & \\
\hline SA & 0.02 & \\
\hline Myocardial infarction & 0.13 & $\uparrow$ \\
\hline Major bleeding from access site & 0.11 & $\leftrightarrow$ \\
\hline Suden cardiac arrest & 0.41 & $\leftrightarrow$ \\
\hline Allergic reaction & 0.01 & $\downarrow$ \\
\hline Artery perforation & 0.24 & $\uparrow$ \\
\hline No reflow & 0.70 & $\uparrow$
\end{tabular}

Structural procedures are a vital part of interventional cardiology in Poland and are mainly performed by certified interventional cardiologists. Following recommendations of the European Society of Cardiology, 22 multidisciplinary Heart Teams involving 57 certified interventional cardiologists were established in all 22 transcathether aortic valve implantation (TAVI) centers. There were 1550 TAVI procedures performed in 22 centers (including 55 from apical access). Evolut/Evolut Pro (Medtronic, USA) was implanted in 893, Sapien 3/Sapien XT (Edwards Lifesciences, USA) in 316, Acurate (Boston Scientific, USA) in 177, Portico (Abbott, USA) in 127, MyValve (Merill, India) in 21, Hydra (Vascular Innovations Company, Thailand) in 10 (only as a part of a clinical study) and Lotus Edge (Boston Scientific, USA) in 6 patients. Moreover, there were 29 procedures of pulmonary valve implantation including Melody (Medtronic, USA) in 18 and Sapien 3/ Sapien XT (Edwards Lifesciences, USA) in 11 patients. The median number of TAVI procedures per center per annum increased to 70 in 2019 (Figure 2). Although the total number of procedures increased by $22 \%$ (year to year), Poland with the number of $40 \mathrm{TAVI}$ procedures per million inhabitatants in 2019 is far below the European

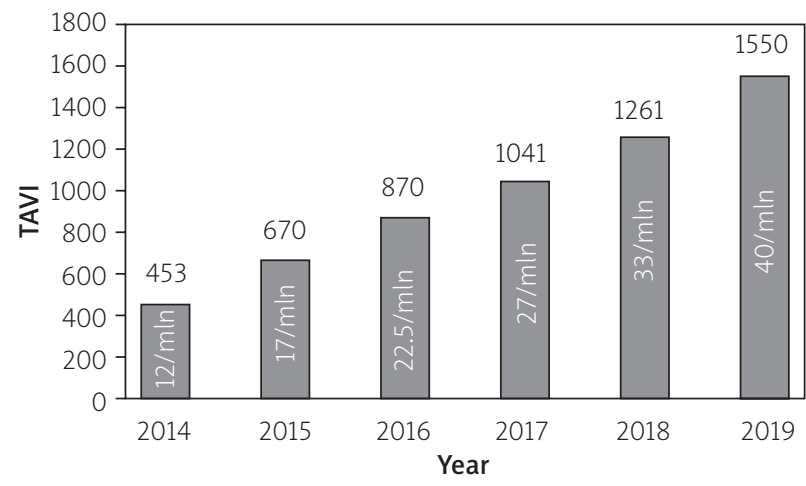

Figure 2. The number of TAVI procedures in Poland (22 centres)
Table III. Additional intracoronary assessment in 2019 during angiography and PCI

\begin{tabular}{lcc} 
Parameter & N & \% change from 2018 \\
\hline FFR & 9916 & $\uparrow 9$ \\
\hline IVUS & 4400 & $\uparrow 21$ \\
\hline OCT & 381 & $\leftrightarrow$
\end{tabular}

average. In 2017 the annual number of TAVI procedures in Germany reached almost 20000 , with the TAVI rate of 250 per million inhabitants [6]. The data presented at the EuroPCR and ESC events in 2019 confirmed an increase of the number Tavi procedures in France from 7,500 in 2014 to 20,000 in 2018 (137\%) with the TAVI penetration of 193 per million inhabitants in 2018 [7]. Then finally Portugal, often compared to Poland, recorded a significant increase of TAVI procedures during the last 5 years from 29 in 2015 to 72 in 2019 per million inhabitants [8]. It is worth mentioning that all three countries participate in the Valve-for-Life Initiative of the EAPCI ESC (Poland and France since 2015 and Portugal since 2017).

MitraClips (Abbott, USA) were implanted in $187 \mathrm{pa}$ tients in 12 centers (including one performing procedures only within clinical trials). Although the number of procedures increased by $26 \%$ (year to year), Poland with about 4.6 MitraClip procedures per million inhabitans is far below the clinical needs (Figure 3).

As many as 597 left atrial appendage closure procedures were performed in 28 centers including 315 Watchman/Watchman FLX (Boston Scientific, USA), 262 Amulet (Abbott, USA) and 20 Lambre (Lifetech, China). The number of LAA closure procedures increased by almost 50\% (year to year).

In summary, we can observe a continuous decreasing trend in both diagnostic and therapeutic percutaneous coronary procedures in comparison to previous years. However, it has to be interpreted cautiously because the data are reported on a voluntary basis. On the other hand, the use of a radial approach, IVUS and ticagrelor in MI pa-

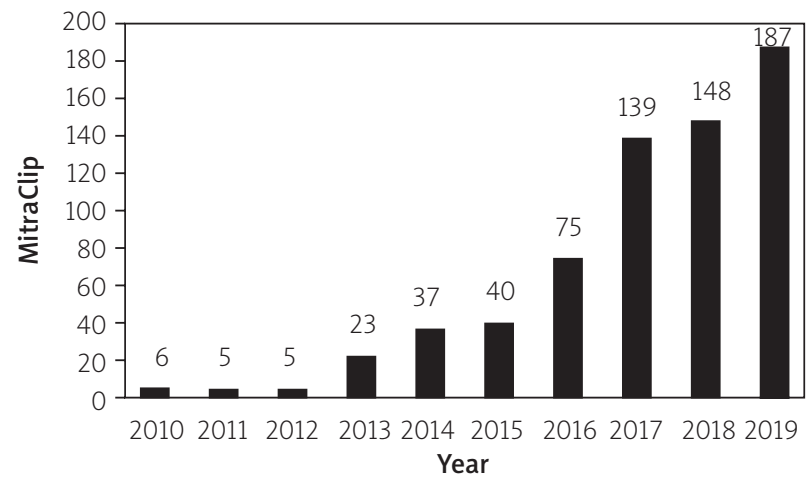

Figure 3. The number of MitraClip procedures in Poland 
tients is well in line with ESC recommendations. Use of the bare-metal stent (BMS) and BVS is almost completely abandoned. The number of structural procedures shows a steady rise in the last years, although it is still limited by reimbursement issues and is far below the European average and current clinical needs in Poland (especially for TAVI and MitraClip).

\section{Conflict of interest}

Wojciech Wojakowski: Medtronic advisory board, Abbott, Edwards, Balton, Moston Scientific, Astra Zeneca - lecture honoraria. Other authors declare no conflict of interest.

\section{References}

1. Dudek D, Siudak Z, Grygier M, et al. Interventional cardiology procedures in Poland in 2018. Summary report of the Association of Cardiovascular Interventions of the Polish Cardiac Society (AISN PTK) and Jagiellonian University Medical College. Adv Interv Cardiol 2019; 15: 391-3.

2. Kleczyński P, Siudak Z, Dziewierz A, et al. The network of invasive cardiology facilities in Poland in 2016 (data from the ORPKI Polish National Registry). Kardiol Pol 2018; 76: 805-7.

3. Zabojszcz M, Januszek R, Siudak Z, et al. Association between the mortality rate and operator volume in patients undergoing emergency or elective percutaneous coronary interventions. Kardiol Pol 2020; 78: 138-46.

4. Tokarek T, Dziewierz A, Plens K, et al. Radial approach expertise and clinical outcomes of percutanous coronary interventions performed using femoral approach. J Clin Med 2019; 8: pii: E1484.

5. Januszek R, Siudak Z, Reczuch K, et al. Current trends and procedural outcomes in the era of rotational atherectomy expansion in Poland in the period 2014-2017 (based on the nationwide ORPKI registry). Adv Interv Cardiol 2019; 15: 158-66.

6. Eggebrecht H., Mehta R. H. Transcatheter aortic valve implantation (TAVI) in Germany: more than 100,000 procedures and now the standard of care for the elderly. Eurolntervention 2019; 14: e1549-52.

7. https://www.escardio.org/Sub-specialty-communities/European-Association-of-Percutaneous-Cardiovascular-Interventions-(EAPCI)/Advocacy/valve-for-life-france.

8. https://www.escardio.org/Sub-specialty-communities/European-Association-of-Percutaneous-Cardiovascular-Interventions-(EAPCI)/Advocacy/valves-for-life-portugal 